\title{
Drug-related problems among hospitalized cancer pain patients: an investigative single-arm intervention trial
}

\author{
Ying-Jie Su ${ }^{1,2 \#}$, Yi-Dan Yan ${ }^{1 \#}$, Wen-Juan Wang ${ }^{3}$, Tao Xu ${ }^{4}$, Zhi-Chun Gu ${ }^{1}$, Yong-Rui Bai ${ }^{2}$, Hou-Wen Lin ${ }^{1}$ \\ ${ }^{1}$ Department of Pharmacy, Renji Hospital, School of Medicine, Shanghai Jiao Tong University, Shanghai, China; ${ }^{2}$ Department of Radiation \\ Oncology, Renji Hospital, School of Medicine, Shanghai Jiao Tong University, Shanghai, China; ${ }^{3}$ Department of Pharmacy, Children's Hospital of \\ Soochow University, Suzhou, China; ${ }^{4}$ Department of Pharmacy, Ningbo First Hospital, Ningbo, China \\ Contributions: (I) Conception and design: YJ Su, YD Yan; (II) Administrative support: ZC Gu, YR Bai, HW Lin; (III) Provision of study materials \\ or patients: YJ Su, YD Yan; (IV) Collection and assembly of data: YJ Su, YD Yan; (V) Data analysis and interpretation: YJ Su, YD Yan, ZC Gu; (VI) \\ Manuscript writing: All authors; (VII) Final approval of manuscript: All authors. \\ \#These authors contributed equally to this work, and should be considered as co-first authors. \\ Correspondence to: Zhi-Chun Gu. Department of Pharmacy, Renji Hospital, School of Medicine, Shanghai Jiaotong University, Shanghai 200127, \\ China. Email: guzhichun213@163.com; Yong-Rui Bai. Department of Radiation Oncology, Renji Hospital, School of Medicine, Shanghai Jiao Tong \\ University, Shanghai 200127, China. Email: baiyongrui@renji.com.
}

Background To evaluate the characteristics of drug-related problems (DRPs) in cancer pain patients, and to identify the impact of pharmacists' intervention in cancer pain associated DRPs.

Methods: In this investigative, single-arm intervention study, clinical pharmacists identified DRPs in cancer pain patients and provided interventions based on medication information, direct patient-pharmacist interview, and ward rounds with multi-disciplinary team (MDT). Types and causes of DRPs, interventions, acceptance and outcome were sorted based on Pharmaceutical Care Network Europe (PCNE) DRP classification V9.0, which includes 3 primary domains for problems, 9 for causes, 5 for interventions, 3 for acceptance, and 4 for DRPs status.

Results: Totally, 42 cancer pain patients were enrolled, and 47 DRPs in 33 (78.6\%) patients were identified by clinical pharmacists. The major type of DRPs was treatment effectiveness $(30 ; 63.8 \%)$ and treatment safety $(17 ; 36.2 \%)$. For the "treatment effectiveness" category, the "effect of drug treatment not optimal" was dominant category $(27 / 30 ; 90 \%)$. A total of 66 DRP causes were identified, and most of DRPs were caused by "drug selection" $(27 ; 40.9 \%)$ and "dose selection" $(16 ; 24.2 \%)$. Within the "drug selection" category, "no or incomplete drug treatment in spite of existing indication" was dominant category $(25 / 27 ; 92.6 \%)$. According to DRPs, 159 interventions were provided by clinical pharmacists and $99.4 \%$ of interventions were accepted by prescribers or patients. Finally, 44 (93.6\%) DRPs were solved.

Conclusions: In cancer pain patients, insufficient pain control mainly caused by inappropriate selection and dosage of analgesics. Clinical pharmacists' interventions dramatically ameliorate these problems and bring about positive effects in cancer pain pharmacotherapy.

Keywords: Drug-related problems (DRPs); cancer pain; clinical pharmacist; pharmacy services; interventions

Submitted Jul 22, 2020. Accepted for publication Nov 17, 2020.

doi: 10.21037/apm-20-1458

View this article at: http://dx.doi.org/10.21037/apm-20-1458

\section{Introduction}

According to the official definition by Pharmaceutical Care Network Europe (PCNE), Drug-Related Problems (DRPs), which are events or circumstances involving pharmacotherapy, potentially or actually interferes with desired health outcomes (1). The World Health Organization (WHO) estimates that more than one half of all medicines exist DRPs in their prescription or 
administration (2). Cancer pain patients are at a significantly increasing risk of DRPs due to the combination of multiple drugs, including but not limited to antineoplastic agents, analgesics, supportive care medications, drugs for adverse effect and complications $(3,4)$. Ignored DRPs are responsible for increased risk of hospital admissions, as well as emergency department visits (2). Furthermore, fatal adverse drug reactions (ADRs), half judged to be related to DRPs (5), are associated with patient's death (6), making an enormous burden on global health care utilization and suggesting a challenge for improvement.

Clinical pharmacists, by virtue of their expertise in managing medication, identifying and resolving complex DRPs, could assist patients to achieve optimal outcome in pharmacotherapy. In the United States, the U.S. surgeon general, Centers for Disease Control and Prevention, and Institute of Medicine have noted that pharmacists are essential members in the health care team (7). In China, clinical pharmacists also play an increasingly critical role in drug therapy, and pay more attention to clinical DRPs $(8,9)$.

Classification of DRPs, as a process indicator of pharmaceutical care outcomes in experimental trials, is necessary for use in research into the incidence, prevalence, and nature of DRPs. In addition, it facilitates pharmacists to describe and assess DRPs in pharmaceutical care practice. To date, more than 20 different types of classification systems for DRPs have been developed. However, these classification systems vary greatly in terms of category size, type, and content, making it difficult for comparison $(2,10)$. Among available classification systems, PCNE, a hierarchical classification system according to types and causes of DRPs, as well as pharmacists' interventions, acceptances and outcomes, is widely used in DRPs description $(1,2,10)$. In prior seven DRPs-related studies in China, four applied the PCNE classification system (11-14), one used the DOCUMENT classification system (15), and the other two categorized the DRPs by researchers themselves rather than any classification system $(8,16)$.

Of late years, DRPs has been growingly studied by pharmacists. Although cancer pain patients are at a significant risk of DRPs, no DRP-related studies currently have been specifically addressed in these patients based on PCNE classification system in China $(9,16)$. In this pharmacist-led study, the PCNE classification system was used to analyze the characteristics of DRPs in cancer pain patients, as well as to identify the impact of pharmacists' intervention in cancer pain associated DRPs. We present the following article in accordance with the STROBE reporting checklist (available at http://dx.doi.org/10.21037/ apm-20-1458).

\section{Methods}

\section{Study design and patients}

This investigative, single-arm intervention study was carried out in a teaching hospital in China (Renji Hospital, School of Medicine, Shanghai Jiaotong University). Because this is an exploratory research, the sample size was not calculated. The group of clinical pharmacists participated in daily ward rounds with the group of physicians in department of radiation oncology, and new admitted cancer patients were eligible for inclusion if they comply with criteria as follows: (I) over 18 years old; (II) confirmed cancer diagnosis; (III) diagnosis of cancer pain by the treating physician; (IV) able to comprehend, speak, and read Chinese. As present trial focused on the pharmacological treatment of cancer pain, patients with invasive pain treatment (e.g., patientcontrolled analgesia or nerve block) were excluded. The study was carried in accordance with the Declaration of Helsinki (as revised in 2013), and was approved by the Ethics Committee of Renji Hospital, School of Medicine, Shanghai Jiaotong University (KY2020-036). All patients have provided written informed consent.

\section{Assessment system of DRPs}

The identified DRPs were characterized using the hierarchical-designed PCNE DRP classification V9.0 (1), with respective codes: three primary domains for different problems (P1-P3), nine primary domains for causes (C1-C9), five primary domains for types of interventions (I0-I4), three primary domains for acceptance of intervention recommendation (A1-A3) and four primary domains for status of the DRPs (O0-O3). Detailed classifications were in subdomains under the primary domains: 7 subdomains for different problems, 43 subdomains for causes, 17 subdomains for types of interventions, 10 subdomains for acceptance, and 7 subdomains for status of the DRPs. In clinical settings, one problem (P) may exist multiple causes (C), leading to more than one intervention (I) or acceptance (A), but leads to only one status of the DRPs (O).

\section{Clinical pharmacist model based on DRPs}

A multi-disciplinary team (MDT), including physicians, 
nurses and clinical pharmacists, was consisted for medical care in the present study. In the group of clinical pharmacists, two junior pharmacists (Y.D.Y. and W.J.W.) participated in the assessment of cancer pain and identification of DRPs, one senior pharmacist-in-charge (Y.J.S.) was responsible for checking DRPs as well as providing intervention and feedback. All the DRPs and subsequent recommendations were provided according to the National Comprehensive Cancer Network Adult Cancer Pain Guidelines (version 1.2018) (17). At the initiation of a cancer pain patient's enrollment, pharmacists provided a comprehensive assessment (including pain characteristics, pain intensity, current analgesic strategy, medication adherence and adverse effects), and offered medication education. Afterwards, reassessments were conducted daily and weekly before and after pain control, respectively. During 28-day follow-up, analgesic efficacy and safety were monitored by face-to-face interview during hospitalization or via telephone after discharge. Due to a 48 -hour window period for patients hospitalized at weekend, examination of these patients' prescriptions was performed within 48 hours from diagnosis of cancer pain. The clinical pharmacists identified and recorded possible DRPs using PCNE DRP classification V9.0. based on daily ward rounds with MDT, patient-pharmacist interview, as well as medication review. Accordingly, intervention proposal to optimize analgesic therapy were provided for physicians.

\section{Typical case}

A typical case was presented to describe the whole process of interventions by clinical pharmacists. A 54-year-old man with urothelial carcinoma of ureter stage IVA was admitted to the department of radiation oncology ward. The patient had a severe distending pain in left lower abdomen, with a property of visceral pain. Subsequent evaluation and intervention of the treatment protocol by clinical pharmacists focused on following issues. Firstly, according to the guidelines, the combination of opioidacetaminophen products should be avoided due to the hepatotoxicity of over-dose acetaminophen. This patient received oxycodone \& acetaminophen tablets as initial analgesic. The DRP was identified as "adverse drug event (possibly) occurring P2.1" and the cause of the DRP was "inappropriate drug according to guidelines/formulary C1.1". Then the pharmacists recommended morphine or oxycodone sustained release tablets as initial analgesic. Secondly, the patient suffered with breakthrough pain after taking oxycodone sustained release tablets, while immediate release morphine tablets were not available. The DRP was identified as "effect of drug treatment not optimal P1.2", caused by "no or incomplete drug treatment in spite of existing indication C1.6". The pharmacists recommended physician to prescribe immediate release morphine tablets for the breakthrough pain. Thirdly, the patient had opioidassociated constipation, but the physician did not notice and take steps. The DRP was also identified as "adverse drug event (possibly) occurring P2.1" and the cause was identified as "no or inappropriate outcome monitoring C9.1". Then the pharmacists advised the physician for prescribing a laxative.

\section{Outcomes and statistical analysis}

A descriptive analysis was conducted on the patient's demographics, clinical characteristics, and initial analgesics. Types, causes and status of DRPs, as well as interventions by pharmacists and acceptance of advices were collected according to PCNE DRP classification. Categorical variables are presented as the number with percentage, and continuous variables are expressed as mean with standard deviation.

\section{Results}

\section{Patient characteristics}

In total, forty-two patients were enrolled between November 2018 and November 2019, and 33 (78.6\%) patients had DRPs that require pharmacists' interventions. Characteristics and initial analgesics of patients are shown in Table 1 . The mean age was 59 years old, the proportion of male was $66.7 \%$ and average education years were 9.31 . The top three types of tumor were esophageal carcinoma (21.4\%), cervical cancer (19.0\%) and lung cancer (19.0\%). The most frequently used initial analgesics were oxycodone sustained-release tablet (50\%), morphine sustained-release tablets $(28.6 \%)$ and tramadol sustained-release tablets (11.9\%).

\section{Identified DRPs}

Finally, 47 DRPs in 33 patients were identified, with an average of 1.4 DRPs per patient (Table 2). The dominant type of DRPs was "treatment effectiveness P1" (30, 63.8\%), followed by "treatment safety P2" (17, 36.2\%). Within the 
Table 1 Sociodemographics, clinical characteristics and initial analgesics

\begin{tabular}{|c|c|c|c|}
\hline Parameters & Total $(n=42)$ & With DRP $(n=33)$ & Without DRP $(n=9)$ \\
\hline Sex, male, n (\%) & $28(66.7)$ & $23(69.7)$ & $5(55.6)$ \\
\hline Age, years, mean $\pm S D$ & $59( \pm 10)$ & $59.2( \pm 10.1)$ & $59.5( \pm 9.5)$ \\
\hline Education completed, years, mean \pm SD & $9.31( \pm 3)$ & $9.31( \pm 3)$ & $9.35( \pm 3.1)$ \\
\hline Esophageal carcinoma & $9(21.4)$ & $9(27.3)$ & $0(0.0)$ \\
\hline Lung cancer & $8(19.0)$ & $7(21.2)$ & $1(11.1)$ \\
\hline Cervical cancer & $8(19.0)$ & $4(12.1)$ & $4(44.4)$ \\
\hline pancreatic cancer & $6(14.3)$ & $6(18.2)$ & $0(0.0)$ \\
\hline \multicolumn{4}{|l|}{ Initial analgesics, n (\%) } \\
\hline Oxycodone sustained-release tablets & $21(50.0)$ & $16(48.5)$ & $5(55.6)$ \\
\hline Morphine sustained-release tablets & $12(28.6)$ & $11(33.3)$ & $1(11.1)$ \\
\hline Tramadol sustained-release tablets & $5(11.9)$ & $2(6.1)$ & $3(33.3)$ \\
\hline Others & $4(9.5)$ & $4(12.1)$ & $0(0.0)$ \\
\hline
\end{tabular}

DRP, drug-related problem.

Table 2 Recognized problems based on PCNE DRP classification tool

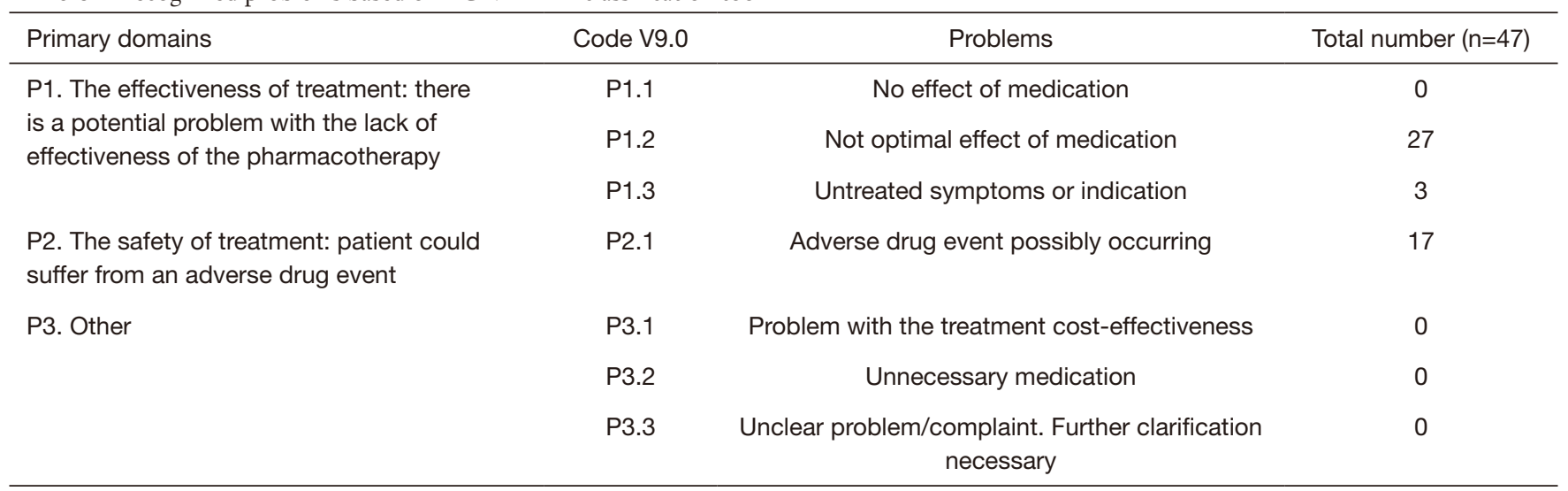

DRP, drug-related problem; PCNE, Pharmaceutical Care Network Europe.

"treatment effectiveness P1" category, the major category was "effect of drug treatment not optimal P1.2" (27/30, 90\%). All of "treatment safety P2" were "adverse drug event (possibly) occurring P2.1".

\section{Causes of DRPs}

Totally, we identified 66 DRP causes (Table 3). The primary cause of DRPs was "drug selection C1" (27; 40.9\%), followed by "dose selection C3" (16; 24.2\%) and "other C9" (14; 21.2\%). Within the "drug selection C1" category, the "no or incomplete drug treatment in spite of existing indication C1.6" was the dominant problem (25/27; 92.6\%). Within the "dose selection C3", "drug dose too low C3.1" was the most common cause of DRPs (10/16; 62.5\%). Almost all of DRPs in "other C9" were caused by "no or 
Table 3 Recognized causes based on PCNE DRP classification tool

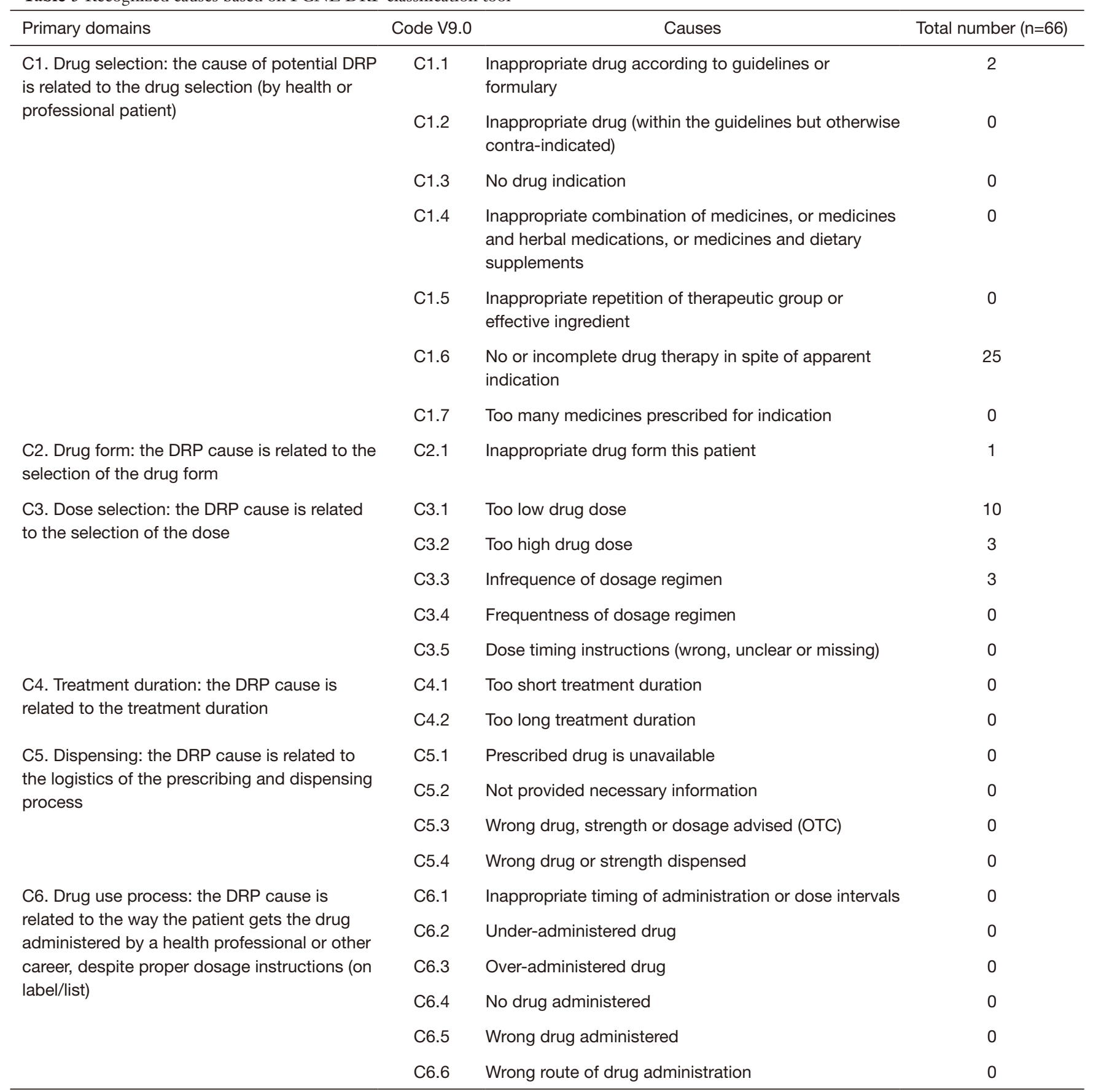

Table 3 (continued) 
Table 3 (continued)

\begin{tabular}{|c|c|c|c|}
\hline Primary domains & Code V9.0 & Causes & Total number $(n=66)$ \\
\hline \multirow{4}{*}{$\begin{array}{l}\text { C7. Patient related: the DRP cause is related } \\
\text { to the patient and his behavior (intentional or } \\
\text { non-intentional) }\end{array}$} & C7.3 & Patient abuses drug & 0 \\
\hline & C7.6 & Patient stores drug improperly & 0 \\
\hline & $\mathrm{C} 7.7$ & Inappropriate timing or dosing intervals & 0 \\
\hline & $\mathrm{C} 7.8$ & Patient takes the drug by a wrong way & 0 \\
\hline \multirow{4}{*}{$\begin{array}{l}\text { C8. Patient transfer related: the DRP cause } \\
\text { can be related to the patients transfer between } \\
\text { cares, or transfer within one care institution. }\end{array}$} & C8.2 & No updated drug list available & 0 \\
\hline & C8.3 & $\begin{array}{l}\text { Discharge or transfer information of incomplete or } \\
\text { missing drug }\end{array}$ & 0 \\
\hline & C8.4 & Insufficient clinical information about the patient & 0 \\
\hline & C8.5 & Patient has not necessary drug at discharge & 0 \\
\hline \multirow[t]{2}{*}{ C9. Other } & C9.1 & No or inappropriate monitoring outcome (e.g., TDM) & 14 \\
\hline & C9.2 & Other specify cause & 1 \\
\hline
\end{tabular}

DRP, drug-related problem; PCNE, Pharmaceutical Care Network Europe.

inappropriate outcome monitoring (incl. TDM) C9.1" (14/15; 93.3\%), among which 9 were not monitored for $\mathrm{ADR}$ and 5 were failure to discontinue analgesics even pain was completely relieved. The other one cause of DRPs in "other cause C9.2" was persistent ADR without remittance.

\section{Proposed and accepted interventions for identified DRPs}

Totally, 159 times of interventions aimed to identified DRPs were offered by clinical pharmacists, with an average of 3.4 interventions per DRP (Table 4). The top two interventions were "at prescriber level I1" (64; 40.3\%) and "at drug level I3" (61; 38.4\%), the other interventions were "at patient level I2" (34; $21.4 \%)$. The most major sub-category was "intervention proposed to prescriber I1.3" (57/64; 89.1\%), "drug started I3.6" (35/61; 57.4\%) and "spoken to family member/caregiver I2.4" (27/34; 79.4\%), respectively. Almost all interventions (158/159; 99.4\%) were "accepted by prescribers or patients A1" (Table 5). Among accepted interventions, 156 (98.7\%) were "accepted and fully implemented A1.1".

\section{Status of the DRPs}

Among 47 identified DRPs, 44 (93.6\%) were "totally solved O1", 1 (2.1\%) was "partially solved O2", and 2 (4.3\%) were "not resolved and intervention not effective O3.3" (Table 6).

\section{Discussion}

\section{Main findings}

Appropriate classification system applied in DRPs related research facilitates to compare results from different studies. To our knowledge, this is the first investigative trial to evaluate DRPs in hospitalized cancer pain patients by 
Table 4 Proposed interventions based on PCNE DRP classification tool

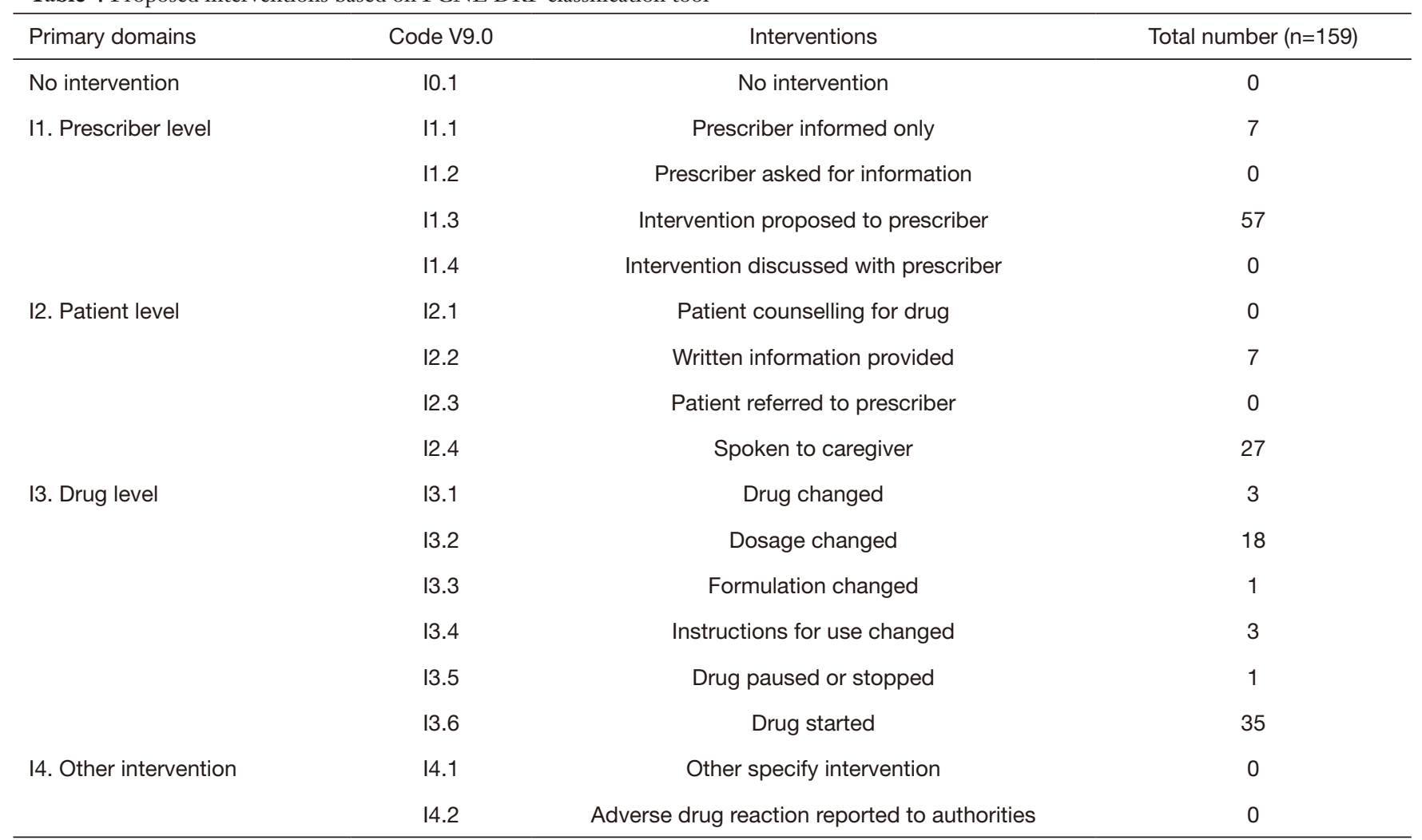

DRP, drug-related problem; PCNE, Pharmaceutical Care Network Europe.

Table 5 Intervention acceptance based on PCNE DRP classification tool

\begin{tabular}{lcccc}
\hline Primary domains & Code V9.0 & Implementation & Total number (n=159) \\
\hline $\begin{array}{l}\text { A1. Intervention accepted by patient } \\
\text { or prescriber }\end{array}$ & A1.1 & Intervention accepted and implemented fully & 156 \\
& A1.2 & Intervention accepted, implemented partially & 1 \\
& A1.3 & Intervention accepted but unimplemented & 1 \\
A2. Intervention not accepted by & A1.4 & Intervention accepted, implementation unknown & 0 \\
patient or prescriber & A2.1 & Intervention unaccepted: not feasible & 0 \\
& A2.2 & Intervention unaccepted: no agreement & 0 \\
A3. Other (no information on & A2.3 & Intervention unaccepted: other specify reason & 1 \\
acceptance) & A3.1 & Intervention unaccepted: unknown reason & 0 \\
\hline
\end{tabular}

DRP, drug-related problem; PCNE, Pharmaceutical Care Network Europe. 
Table 6 DRP Status based on PCNE DRP classification tool

\begin{tabular}{lccc}
\hline Primary domains & Code V9.0 & Outcome of intervention & Total number $(\mathrm{n}=47)$ \\
\hline O0. Unknown & O0.1 & DRP status unknown & 0 \\
O1. Solved & 01.1 & DRP totally solved & 44 \\
O2. Partially solved & O2.1 & DRP partially solved & 1 \\
O3. Not solved & O3.1 & DRP not solved, lack of cooperation of patient & 0 \\
& O3.2 & DRP not solved, lack of cooperation of prescriber & 0 \\
& O3.3 & DRP not solved, intervention not effective & 0 \\
\hline
\end{tabular}

DRP, drug-related problem; PCNE, Pharmaceutical Care Network Europe.

a mature classification systems in China, and to assess the impact of pharmacists' intervention in DRPs. The present study revealed that analgesic DRPs in cancer pain patients, which mainly derived from inappropriate drug selection and dosage, were common and can be resolved by pharmacists' interventions.

\section{The overall DRPs in cancer pain patients}

The DRPs are extremely common in cancer pain patients. Our results showed that $78.6 \%$ (33/42) patients had DRPs and required pharmacists interventions. A previous research, which was conducted in Union Hospital in China, reported that the incidence of DRPs was up to $110.77 \%$ for the use of analgesics in cancer pain patients (16). Semerjian et al. also conducted a retrospective study at an academic medical center in Los Angeles, and indicated that $98.7 \%$ of pain clinic patients had one or more DRPs (18). We recognized that the proportion of DRPs in previous two studies was higher than that in ours. However, incidence of DRPs reported in cancer patients was similar with ours. The pharmacists in Turkey reported that majority of patients (83.2\%) in hospitalized oncology had at least one DRP (3). Besides, a Netherlandish scholar conducted a study in elderly patients ( $\geq 65$ years) with more than five chronic drugs in oncology clinic, and revealed that $78 \%$ of patients had DRPs (4). To sum up, the DRP incidence found in our study was similar to that reported in cancer patients, but lower than that reported in cancer pain patients. It is possible that clinical pharmacists have corrected some of potential DRPs during their 10-year pharmacy services in department of radiation oncology. For instance, physicians in our MDT preferred to choose oral morphine or oxycodone rather than fentanyl patch as initial analgesics for opioid-naïve patients.

\section{The type and cause of DRPs}

As for the types of DRPs, the most problem was "effect of drug treatment not optimal P1.2", a subdirectory under the "treatment effectiveness P1" category. In terms of the causes, approximately $38 \%$ of DRPs $(25 / 66)$ were caused by "no or incomplete drug treatment in spite of existing indication C1.6", a subdirectory belonging to the "drug selection C1" category. Besides, only 10.6\% (7/66) DRPs were caused by "patient uses/takes less drug than prescribed or does not take the drug at all C7.1". Therefore, inappropriate selection and dosage of analgesics might be major reason for most DRPs of "treatment effectiveness P1" in patients with cancer pain. However, the results were different from the study in Wuhan, China (16). They divided pharmacotherapeutic DRPs into 12 types, and reported that nonadherence or missed doses $(27.69 \%)$ was the major cause of DRPs, followed by inappropriate opioid selection $(22.56 \%)$ and inappropriate dosage (16.41\%). In our study, medication education was carried out frequently for cancer pain patients during patient-pharmacist interview, resulting in a low proportion of "nonadherence or missed doses" in the causes of DRPs. For above evidences, medication education by clinical pharmacists is important in the process of cancer pain pharmacotherapy.

\section{The acceptance of pharmacists' intervention of DRPs}

According to previous studies, the acceptance of pharmacists' interventions was satisfactory. A Turkish study reported that acceptance rate of intervention proposals was $93 \%$ in hospitalized oncology patients, and 
$90.9 \%$ of the identified problems were totally solved (3). In addition, two Chinese researches in hospitalized respiratory patients revealed that a total of $91.0-96.2 \%$ of interventions for DRPs were accepted, and 81.9-91.6\% DRPs were resolved $(11,14)$. In consistent with previous results, almost all interventions (99.4\%) were accepted by prescribers or patients and $93.6 \%$ of DRPs were totally solved in our study, indicating the necessity and popularity of pharmaceutical care for physicians and patients.

\section{Strengths and limitations}

This is the first investigative trial to evaluate DRPs in hospitalized patients with cancer pain in China based on a mature classification system, and to assess the impact of pharmacists' interventions in DRPs. Meanwhile, our study had explored clinical pharmacist's positive role in the treatment of cancer pain patients. Ten-year work of pharmacists in present study enabled their abundant experience on handling multiple links of cancer pain therapy. Their meticulous and professional pharmaceutical care for cancer pain patients has filled in the gaps of physicians' pharmacotherapy. Several limitations need to be addressed. First, this is a preliminary trial conducted in single center with a relatively small sample size. Therefore, this promising model of pharmacy service must be validated in further studies with a larger population. Second, no control group was included in present study. Thus, we compared results with those in analogous study in China and other countries. Subsequent studies involved control group are necessary to be carried out. Third, only DRPs was used as a clinical outcome indicator, clinical outcomes behind the DRPs were not available. Fourth, comorbid medical conditions were not addressed in present study, and will be included in our future studies. Finally, the pharmacist-led DRPs intervention model was carried out in cancer pain patients, and its effect in other indications needs to be verified.

\section{Conclusions}

DRPs are common in cancer pain patients and insufficient pain control mainly caused by inappropriate selection and dosage of analgesics. Encouragingly, interventions by clinical pharmacists dramatically ameliorate these problems and bring about positive effects in cancer pain pharmacotherapy.

\section{Acknowledgments}

The authors sincerely thank the physicians, nurses and administrators in the treatment team.

Funding: This study was supported by Research Funds of Shanghai Health and Family Planning commission (20184Y0022), Cultivation fund of clinical research of Renji hospital (PY2018-III-06), Clinical Pharmacy Innovation Research Institute of Shanghai Jiao Tong University School of Medicine (CXYJY2019ZD001, CXYJY2019QN004) and Shanghai "Rising Stars of Medical Talent" Youth Development Program - Youth Medical Talents - Clinical Pharmacist Program [SHWJRS (2019) 072; SHWRS (2020) 087].

\section{Footnote}

Reporting Checklist: The authors have completed the STROBE reporting checklist. Available at http://dx.doi.org/10.21037/ apm-20-1458

Data Sharing Statement: Available at http://dx.doi. org/10.21037/apm-20-1458

Peer Review File: Available at http://dx.doi.org/10.21037/apm20-1458

Conflicts of Interest: All authors have completed the ICMJE uniform disclosure form (available at http://dx.doi. org/10.21037/apm-20-1458). The authors have no conflicts of interest to declare.

Ethical Statement: The authors are accountable for all aspects of the work in ensuring that questions related to the accuracy or integrity of any part of the work are appropriately investigated and resolved. The study was conducted in accordance with the Declaration of Helsinki (as revised in 2013). This study was approved by Ethics Committee of Renji Hospital, School of Medicine, Shanghai Jiaotong University (KY2020-036). Written informed consent was obtained from the patients for publication of this study.

Open Access Statement: This is an Open Access article distributed in accordance with the Creative Commons Attribution-NonCommercial-NoDerivs 4.0 International License (CC BY-NC-ND 4.0), which permits the noncommercial replication and distribution of the article with 
the strict proviso that no changes or edits are made and the original work is properly cited (including links to both the formal publication through the relevant DOI and the license). See: https://creativecommons.org/licenses/by-nc-nd/4.0/.

\section{References}

1. Pharmaceutical Care Network Europe (PCNE) DRP Classification V9.00. Available online: https://www.pcne. org/upload/files/410_PCNE_classification_V9-0m.pdf

2. Plácido AI, Herdeiro MT, Morgado M, et al. Drug-related Problems in Home-dwelling Older Adults: A Systematic Review. Clin Ther 2020;42:559-72.e14.

3. Umar RM, Apikoglu-Rabus S, Yumuk PF. Significance of a clinical pharmacist-led comprehensive medication management program for hospitalized oncology patients. Int J Clin Pharm 2020;42:652-61.

4. Vrijkorte E, de Vries J, Schaafsma R, et al. Optimising pharmacotherapy in older cancer patients with polypharmacy. Eur J Cancer Care (Engl) 2020;29:e13185 .

5. Buajordet I, Ebbesen J, Erikssen J, et al. Fatal adverse drug events: the paradox of drug treatment. J Intern Med 2001;250:327-41.

6. DiPoto JP, Buckley MS, Kane-Gill SL. Evaluation of an automated surveillance system using trigger alerts to prevent adverse drug events in the intensive care unit and general ward. Drug Saf 2015;38:311-7.

7. American College of Clinical Pharmacy, McBane SE, Dopp AL, et al. Collaborative drug therapy management and comprehensive medication management-2015. Pharmacotherapy 2015;35:e39-50.

8. Zhai XB, Gu ZC, Liu XY. Clinical pharmacist intervention reduces mortality in patients with acute myocardial infarction: a propensity score matched analysis. Eur J Hosp Pharm 2019;26:248-52.

Cite this article as: Su YJ, Yan YD, Wang WJ, Xu T, Gu ZC, Bai YR, Lin HW. Drug-related problems among hospitalized cancer pain patients: an investigative single-arm intervention trial. Ann Palliat Med 2021;10(2):2008-2017. doi: 10.21037/apm-201458
9. Chen J, Lu XY, Wang WJ, et al. Impact of a clinical pharmacist-led guidance team on cancer pain therapy in China: a prospective multicenter cohort study. J Pain Symptom Manage 2014;48:500-9.

10. Basger BJ, Moles RJ, Chen TF. Development of an aggregated system for classifying causes of drug-related problems. Ann Pharmacother 2015;49:405-18.

11. Zhu Y, Liu C, Zhang Y, et al. Identification and resolution of drug-related problems in a tertiary hospital respiratory unit in China. Int J Clin Pharm 2019;41:1570-7.

12. Wang HY, Yeh MK, Ho CH, et al. Cross-sectional investigation of drug-related problems among adults in a medical center outpatient clinic: application of virtual medicine records in the cloud. Pharmacoepidemiol Drug Saf 2017;26:71-80.

13. Qu C, Meng L, Wang N, et al. Identify and categorize drug-related problems in hospitalized surgical patients in China. Int J Clin Pharm 2019;41:13-7.

14. Li Q, Qu HJ, Lv D, et al. Drug-related problems among hospitalized patients with COPD in mainland China. Int J Clin Pharm 2019;41:1507-15.

15. Yang J, Meng L, Liu Y, et al. Drug-related problems among community-dwelling older adults in mainland China. Int J Clin Pharm 2018;40:368-75.

16. Liu J, Wang C, Chen X, et al. Evaluation of pharmacist interventions as part of a multidisciplinary cancer pain management team in a Chinese academic medical center. J Am Pharm Assoc (2003) 2020;60:76-80.

17. National Comprehensive Cancer Network Adult Cancer Pain Guidelines (version 1.2018). Available online: https:// www.nccn.org/professionals/physician_gls/default. aspx\#supportive

18. Semerjian M, Durham MJ, Mirzaian E, et al. Clinical Pharmacy Services in a Multidisciplinary Specialty Pain Clinic. Pain Pract 2019;19:303-9. 